\title{
Prognostic Value of Programmed Cell Death 1 Ligand-1 (PD-L1) or PD-1 Expression in Patients with Osteosarcoma: A Meta-Analysis
}

\author{
Xin Huang1*, Weiyue Zhang2* ${ }^{2 *}$ Zhicai Zhang1, Deyao Shi ${ }^{1}$, Fashuai Wu1 ${ }^{1}$ Binlong Zhong1, Zengwu Shao ${ }^{\circledR}$ \\ 1. Department of Orthopedics, Union Hospital, Tongji Medical College, Huazhong University of Science and Technology, Wuhan 430022, China. \\ 2. Department of Endocrinology, Union Hospital, Tongji Medical College, Huazhong University of Science and Technology, Wuhan 430022, China. \\ * Equal contributors. \\ $\square$ Corresponding author: Zengwu Shao M.D., Ph.D., Department of Orthopedics, Union Hospital, Tongji Medical College, Huazhong University of Science and \\ Technology, Wuhan 430022, China. E-mail: szwproa@163.com \\ (C) Ivyspring International Publisher. This is an open access article distributed under the terms of the Creative Commons Attribution (CC BY-NC) license \\ (https://creativecommons.org/licenses/by-nc/4.0/). See http://ivyspring.com/terms for full terms and conditions.
}

Received: 2018.01.18; Accepted: 2018.04.29; Published: 2018.06.21

\begin{abstract}
Purpose: Programmed cell death 1 ligand-1 (PD-LI) and PD-1 as prognostic biomarkers have spurred considerable interest in several types of malignant tumors. In the present meta-analysis, we aimed to elucidate the clinicopathological and prognostic values of PD-L1/PD-1 in osteosarcoma.

Methods: We systematically searched PubMed, Web of Science, EMBASE, Scopus, CBM and the Cochrane Library databases up to March 3, 2018. Eligible studies assessing the relationship between PD-L1 or PD-1 expression and clinicopathological and prognostic outcomes in osteosarcoma were incorporated. Pooled relative risks (RRs) and $95 \%$ confidence intervals ( $\mathrm{Cls}$ ) were used to estimate the outcomes.

Results: Eight studies involving 413 patients were incorporated into our meta-analysis. Pooled results showed that PD-L1/PD-1 overexpression was significantly associated with metastasis $(R R=$ $1.54,95 \% \mathrm{Cl}: 1.12-2.11, \mathrm{p}=0.008)$ in osteosarcoma. Furthermore, osteosarcoma patients exhibited a remarkably higher total mortality risk $(R R=1.86,95 \% \mathrm{Cl}: 1.09-3.17, \mathrm{p}=0.021)$ with PD-L1/PD-1 overexpression. However, no significant reduced overall survival rate $(\mathrm{RR}=0.70,95 \% \mathrm{Cl}: 0.46-1.07$, $\mathrm{P}=0.103)$ was detected in the study.
\end{abstract}

Conclusion: Our meta-analysis indicates that PD-L1/PD-1 may serve as an important biomarker for adverse clinicopathologic features and poor prognosis in patients with osteosarcoma.

Key words: Programmed death ligand-1; Osteosarcoma; Prognosis; Meta-analysis.

\section{Introduction}

Osteosarcoma is one of the most common primary malignant bone tumors, especially in children and young adults. Although surgery in combination with chemotherapy has increased the disease-free survival rate to over 60\% [1], limited effects have been shown to protect patients against the recurrent or metastatic osteosarcoma [2]. Therefore, more effective therapeutic strategies are warranted for the treatment of osteosarcoma.

Programmed death ligand-1 (PD-L1) is expressed by most cell types including cancer cells
[3-5]. It is speculated that PD-L1 plays a pivotal role in delivering an inhibitory signal to programmed cell death 1 (PD-1) expressing T cells, resulting in immune system impairment [6]. Recent evidence strongly suggests that the activation of the PD-L1/PD-1 pathway allows tumors to adopt an immune evasion mechanism [7, 8]. Additionally, PD-L1 expression is reported to be significantly in correlation with poor prognosis in cancer $[9,10]$. Blocking the PD-L1/PD-1 pathway induces significant objective responses and prolonged stabilization of the disease in patients with 
cancer [11]. The immunomodulatory therapies targeting the PD-L1/PD-1 immune-checkpoint pathway promote remarkable anti-tumor immunity and have shown considerable success in a subset of solid tumors, including melanoma, lung cancer, and head and neck carcinomas [12].

Although the PD-L1/PD-1 pathway is heavily targeted for anticancer drug discovery, the prognostic and therapeutic roles of PD-L1/PD-1 in osteosarcoma remain largely unknown. In this study, we aimed to perform a meta-analysis to determine the clinicopathological and prognostic values of PD-L1/PD-1 in osteosarcoma.

\section{Methods}

\section{Data search strategy}

We searched PubMed, Web of Science, EMBASE, Scopus, CBM and the Cochrane Library databases up to March 3, 2018. A comprehensive search strategy was developed based on the following terms: (1) PD-L1, programmed death ligand-1, B7-H1, programmed cell death 1, PD-1 and (2) osteosarcoma, osteogenic sarcoma. We additionally hand-searched the references of relevant articles and contacted investigators of certain studies when necessary.

\section{Inclusion and exclusion criteria}

Studies were taken into account when they satisfied the following inclusion criteria: (a) patients had a pathological diagnosis of osteosarcoma; (b) the correlation between PD-L1/PD-1, clinicopathological features, and prognosis was discussed. Exclusion criteria were as follows: (a) literatures not pertinent to PD-L1/PD-1 or osteosarcoma; (b) similar studies from the same author as well as multiple duplicate data in the different works; or (c) animal experiments, case reports, correspondences, reviews, expert opinions, letters, talks, or effect estimates from conference abstracts when a full-published study was not available.

\section{Data extraction and quality assessment}

Two investigators (XH, WYZ) evaluated the eligibility of all retrieved studies and extracted the relevant data independently. Extracted databases were then cross-checked between the two authors to rule out any discrepancy. Disagreement was resolved by consulting with a third investigator (ZWS). The following data of each collected studies were extracted independently: author, year of publication, patient number, country, detection method, cut-off values for the positive or high expression of PD-L1/PD-1, and duration of follow-up. The Cochrane Collaboration risk of bias tool was used to assess risk of bias. Our investigation process was in accordance with the Preferred Reporting Items for Systematic Reviews and Meta-Analyses (PRISMA) statement.

\section{Statistical analysis}

The statistical analysis was performed using the Review Manager (RevMan) software version 5.3 and STATA 14. Estimates were summarized as relative risks (RRs) with $95 \%$ confidence interval (CI) for each study. The between-study heterogeneity was evaluated by using the chi-square test and the $\mathrm{I}^{2}$ statistic. An $\mathrm{I}^{2}$ value of $>50 \%$ of the $\mathrm{I}^{2}$ statistic was considered to indicate significant heterogeneity [13]. When a significant heterogeneity existed across the included studies, a random effects model was used for the analysis. Otherwise, the fixed effects model was used. Subgroup analyses were performed to detect the source of heterogeneity. We further conducted sensitivity analyses to substantiate the stability of results and detect the potential source of heterogeneity. Publication bias was evaluated qualitatively by inspecting funnel plots and quantitatively through the Begg's and Egger's test. A two-tailed P-value $<0.05$ implies a statistically significant publication bias.

\section{Results}

\section{Search results}

The study selection process is illustrated in Figure 1. A total of 274 potential articles were identified from the databases search. Among these articles, 230 were excluded after abstract review, leaving 44 articles for the full-text review. In the review, 36 studies were excluded for the reasons as follows: eighteen were eliminated because they were irrelevant to PD-L1/PD-1 or osteosarcoma, four studies were of no relevant outcomes reported, three studies were of reviews, seven studies involved non-human experiments, and four studies were excluded because of insufficient data for analysis. Finally, eight studies with a total of 413 patients that met the inclusion criteria were included in this meta-analysis.

\section{Study selection and characteristics}

Baseline characteristics of the included studies are presented in Table 1 . The publication years of the eligible studies ranged from 2014 to 2017, and the number of patients in each study ranged from 13 to 107. Additionally, the PD-L1/PD-1 expression levels were measured in tumor tissues in all eight studies by immunochemical staining (IHC). As indicated in Table 1, each article had a specific cut-off value, which consequently influenced the positive rates of PD-L1/PD-1 overexpression. Moreover, the mean 


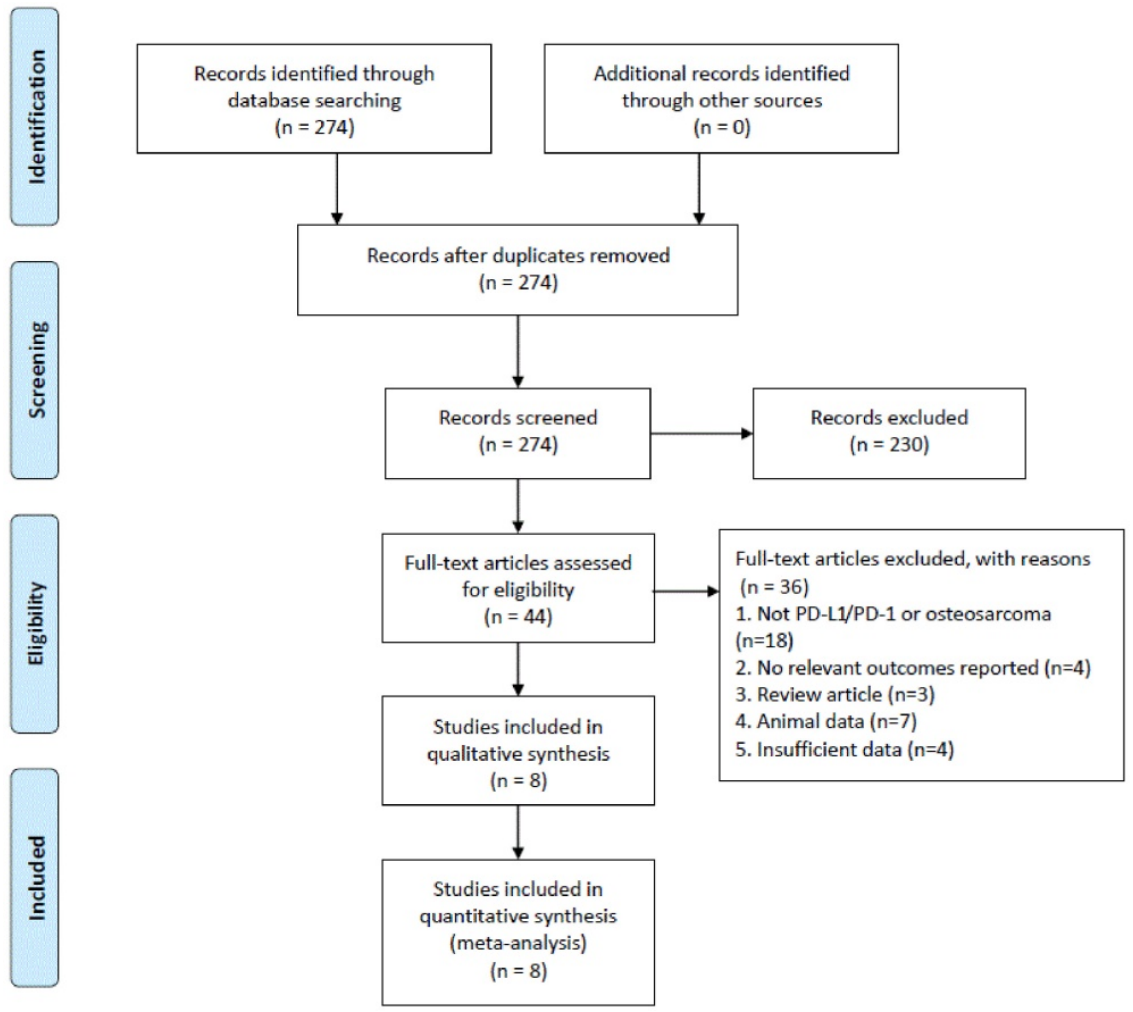

Figure 1. Flowchart of the study selection process.

duration of follow-up after surgery ranged from 25 to 96 months.

\section{PD-L1/PD-1 expression and clinicopathological features}

In the present study, we assessed the relationship between positive/higher PD-L1 expression and clinicopathological features of osteosarcoma patients (Table 2). Metastasis of osteosarcoma was reported in seven studies (Figure $2)$. Due to the absence of heterogeneity $\left(\mathrm{I}^{2}=0.0 \%, \mathrm{p}=\right.$
0.801), the fixed-effect model was adopted, which indicated a pooled RR of 1.54 (95\% CI: 1.12-2.11, $\mathrm{p}=$ 0.008). Therefore, positive/higher PD-L1 expression was significantly associated with increased metastasis in patients with osteosarcoma. However, no significant relationship was observed between PD-L1 overexpression and other clinical characteristics such as gender, tumor size, differentiation of high grade, high tumor stage and response to chemotherapy in osteosarcoma due to insufficient data.

Table 1. Main characteristics of the studies included in this meta-analysis.

\begin{tabular}{|c|c|c|c|c|c|c|c|c|c|}
\hline Study & Year & Patient source & Cases & $\begin{array}{l}\text { PD-L1/PD-1+ } \\
\text { patients (\%) }\end{array}$ & $\begin{array}{l}\text { Follow up } \\
\text { (months) }\end{array}$ & Method & Antibody type & $\begin{array}{l}\text { Antibody } \\
\text { dilution }\end{array}$ & Cutoff value \\
\hline Costa Arantes DA [14] & 2017 & Brazil & 13 & $69.2 \%$ & 32 & IHC & Monoclonal & 1: 400 & IRS $>2$ a \\
\hline Sundara YT [15] & 2017 & Netherlands & 25 & $60 \%$ & 56 & $\mathrm{IHC}$ & Monoclonal & 1: 400 & $\begin{array}{l}\geq 1 \% \text { of tumour cells or } \\
\text { immune cells }\end{array}$ \\
\hline Lussier DM [16] & 2015 & USA & 16 & $75.0 \%$ & NA & $\mathrm{IHC}$ & Monoclonal & $1: 200$ & $>10$ cells / field of view \\
\hline Shen JK [17] & 2014 & USA & 38 & $27.0 \%$ & 36 & $\begin{array}{l}\text { IHC, } \\
\text { qRT-PCR }\end{array}$ & NA & NA & $\begin{array}{l}\text { By IHC, total score } \geq 2 \mathrm{~b} ; \text { By } \\
\text { qRT-PCR, total score }>2-\log \text { c }\end{array}$ \\
\hline Liao YF [18] & 2017 & USA & 72 & $80.6 \%$ & 52 & $\mathrm{IHC}$ & Monoclonal & $1: 50$ & Total score $\geq 2 \mathrm{~b}$ \\
\hline Koirala P [19] & 2016 & USA & 107 & $16.8 \%$ & 25 & $\mathrm{IHC}$ & Monoclonal & $1: 50$ & $>1 \%$ the tumor volume \\
\hline Zheng W [20] & 2015 & China & 56 & $39.3 \%$ & NA & $\mathrm{IHC}$ & Monoclonal & NA & NA \\
\hline Palmerini E [21] & 2016 & Italy & 86 & $14.0 \%$ & 96 & $\mathrm{IHC}$ & NA & NA & NA \\
\hline
\end{tabular}

IHC, immunohistochemistry; IRS, immunoreactive score; qRT-PCR: quantitative real time polymerase chain reaction; NA, not available.

a The IRS was calculated by multiplying a score of staining percentage to another score of staining intensity. The area of staining was scored as 0 (no tumor cells stained), 1 (< $25 \%$ of cells stained), 2 ( $\geq 25 \%$ of cells stained). Staining intensity was graded as 0 (no staining), 1 (weak staining), 2 (moderate staining), 3 (strong staining).

$\mathrm{b}$ Total score was calculated by PD-L1 staining intensity which was graded into four groups: no staining $(0)$, weak staining $(1+)$, moderate staining $(2+)$, and intense staining $(3+)$.

c Absolute PD-L1 expression was normalized to that of housekeeping gene $\beta$-actin and categorized by log-transcript detection: low=1-log, intermediate=2-log, and high=3and 4-log. 
Table 2. Relationship between PD-L1/PD-1 expression and the clinicopathological features.

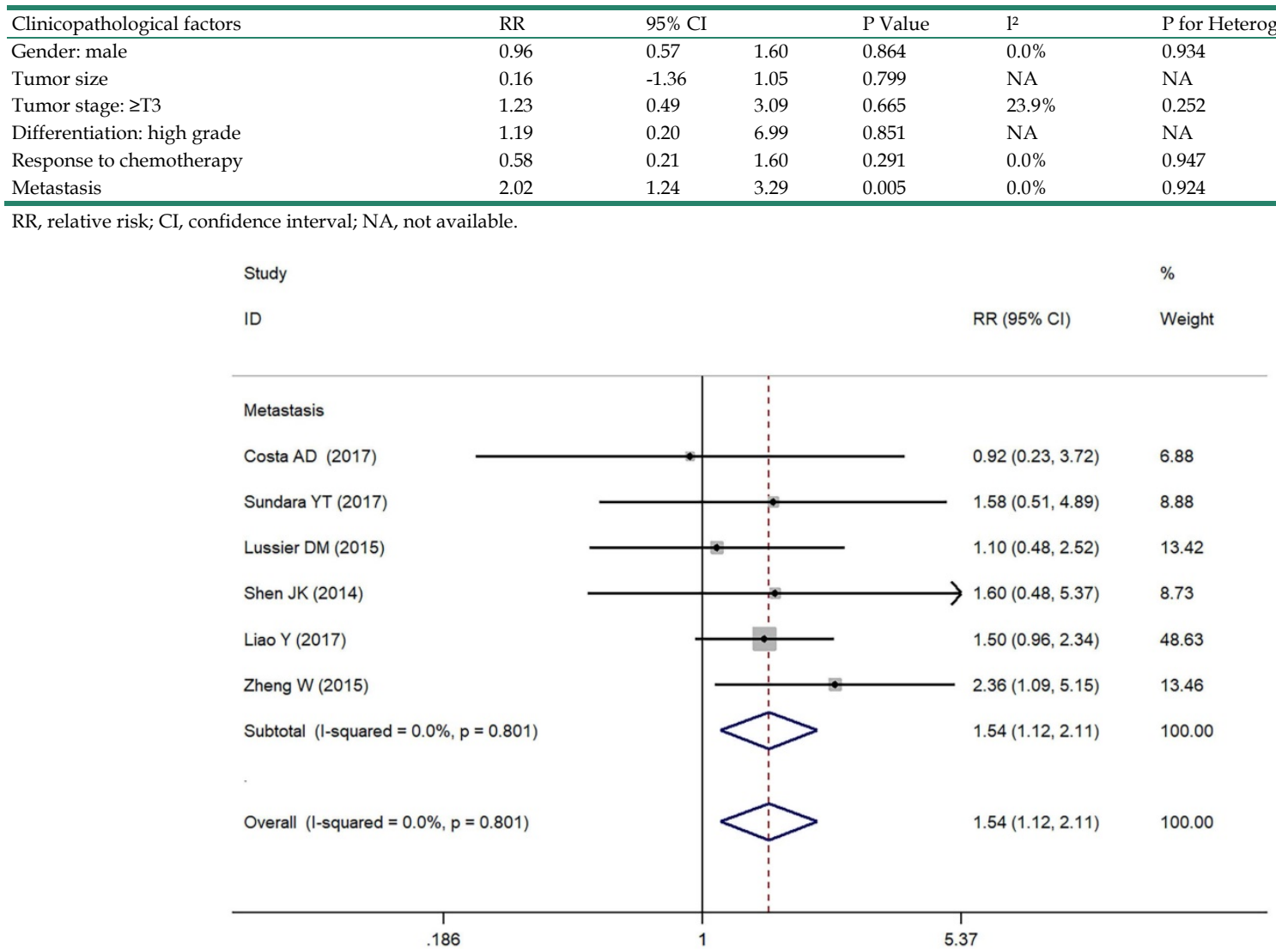

Figure 2. Forest plots for lymph node metastasis of PD-L1/PD-1 in osteosarcoma.

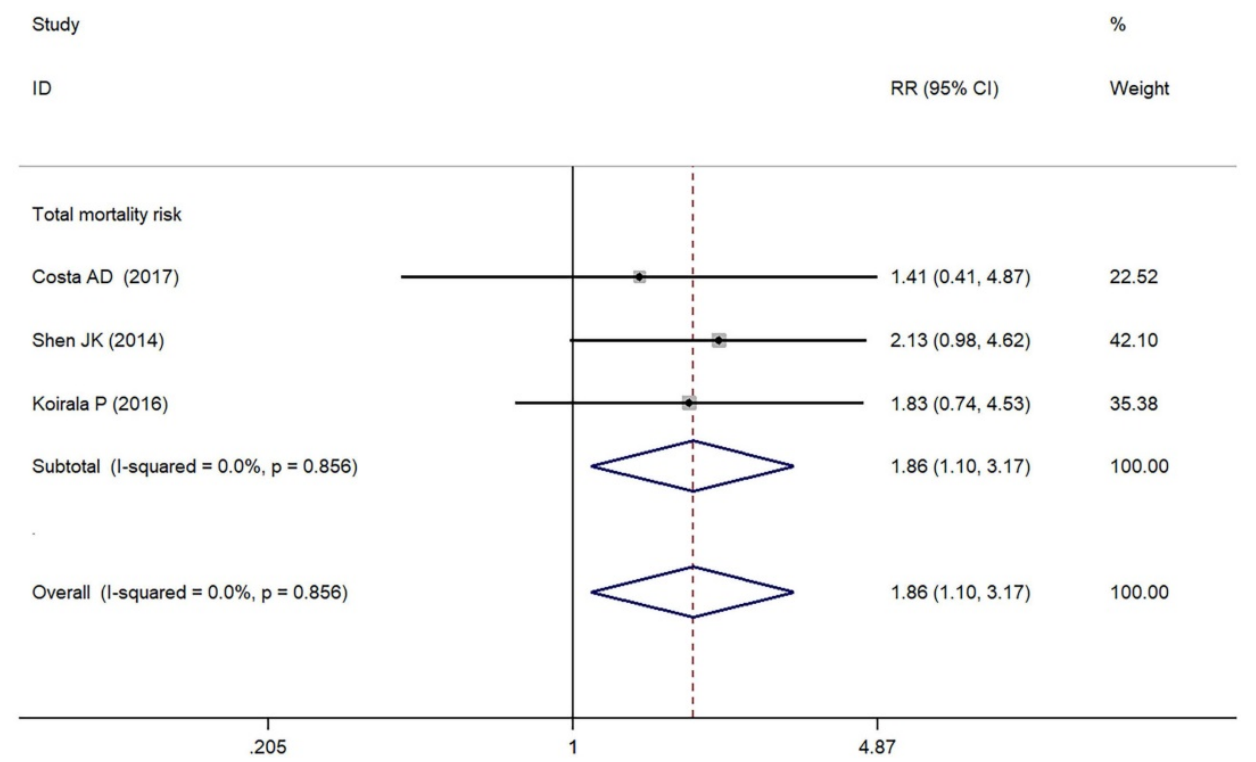

Figure 3. Forest plots for total mortality risk of PD-L1/PD-1 in osteosarcoma.

\section{PD-L1/PD-1 expression and prognostic values}

As depicted in Figure 3, PD-L1/PD-1 overexpression was significantly associated with increased total mortality risk among osteosarcoma patients with the pooled RR 1.86 (95\% CI: 1.09-3.17, p $=0.021)$, and the fixed-effect model was adopted in terms of no significant heterogeneity among the studies $\left(\mathrm{I}^{2}=0.0 \%, \mathrm{p}=0.856\right)$. Compared with the negative/lower PD-L1/PD-1 expression, overall survival decreased $(\mathrm{RR}=0.70,95 \% \mathrm{CI}$ : 0.46-1.07, $\mathrm{p}=$ 0.103 ) in the positive/higher PD-L1/PD-1 expression group. However, the difference was not significant. No heterogeneity among the studies $\left(\mathrm{I}^{2}=0.0 \%, \mathrm{p}=\right.$ 


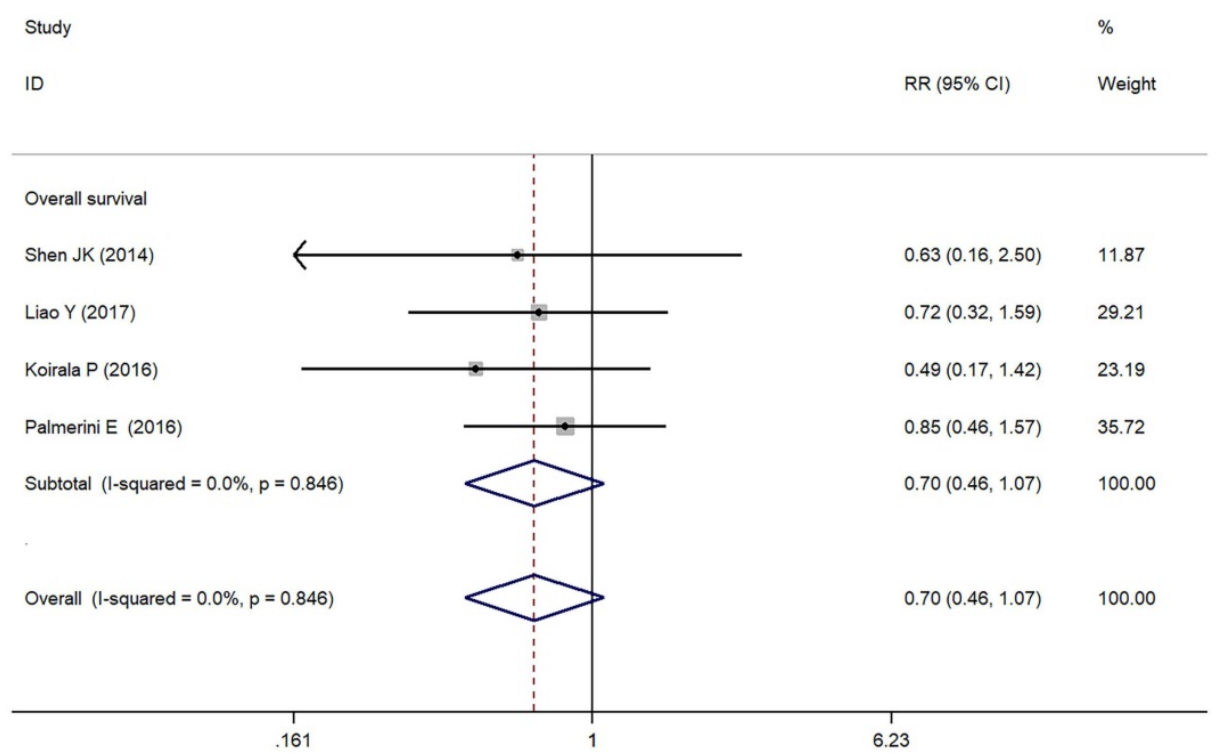

Figure 4. Forest plots for overall survival of PD-L1/PD-1 in osteosarcoma.

0.846) was found and the fixed-effect model was adopted (Figure 4).

\section{Quality assessment}

The quality assessment of each included publication is assessed by the Cochrane Collaboration risk of bias tool (Figure S1A, Figure S1B). In summary, the involved articles were considered with low risk of bias according to the Cochrane collaboration's tool.

\section{Publication bias}

The funnel plot did not indicate any evidence of publication bias in this analysis (Figure S2). No evidence of publication bias was observed from Begg's funnel plot $(p=1.000)$ (Figure S3) and Egger's test $(p=0.863)$ (Figure $S 4)$. To sum up, the possibility of publication bias could be excluded.

\section{Discussion}

The present study revealed a significant association between positive/higher PD-L1/PD-1 expression and clinicopathological and prognostic significance in patients with osteosarcoma. Compared with negative/lower PD-L1/PD-1 expression, PD-L1/PD-1 overexpression for osteosarcoma was significantly associated with increased metastasis, with a tendency for higher total mortality risk and poorer overall survival. Our meta-analysis suggests that PD-L1/PD-1 overexpression predicts poor prognosis and adverse clinicopathologic features of osteosarcoma, which may facilitate the better management of osteosarcoma patients.

It is well-known that PD-1 and its ligand PD-L1 are main mechanisms for tumor immune tolerance and escaping immune surveillance [22]. PD-1 is a cell-surface receptor expressed on subsets of $\mathrm{T}$ and $\mathrm{B}$ lymphocytes as well as on other immune cells. Furthermore, abundant evidences have indicated that PD-1 is a co-inhibitory receptor that negatively regulates T-cell function [23]. Meanwhile, PD-L1, as a transmembrane protein, is expressed on tumorinfiltrating lymphocytes (TILs), antigen present cells (APCs) and tumor cells of cancer including breast, lung, prostate and so on. Based on the study of Shen et al. [17], PD-L1 mRNA and protein expression were also detected in human osteosarcoma samples and in cell lines.

In our study, metastasis of osteosarcoma was reported in seven eligible studies. Previous studies have detected overwhelming evidence about the role of PD-L1 in metastatic osteosarcoma. Utilizing quantitative real-time PCR (qRT-PCR), PD-L1 mRNA expression had positive correlation with TILs, which is regarded as a marker of metastasis [17]. Subsequently, with the method of immunofluorescence (IF), PD-L1 was reported to be specially expressed in metastatic tissues rather than in primary specimens [16]. In accordance with the previous findings, our study strengthened the observation that PD-L1 expression was remarkably associated with metastasis in osteosarcoma. Moreover, by CRISPR/Cas9 system, PD-L1 may play a role in regulating osteosarcoma cell growth and drug resistance to doxorubicin and paclitaxel [18]. However, there was no significant relationship between PD-L1 expression and other clinical characteristics of osteosarcoma. The drug resistance and tumorigenicity of osteosarcoma were supposed to be partly attributed to PD-L1 overexpression, which thereby might be a potential target in clinical treatment [18]. 
Our study also demonstrated that PD-L1/PD-1 may be a significant indicator of poor prognosis for patients with osteosarcoma. The PD-L1/PD-1 complex transmitted an inhibitory signal, which reduced the proliferation of $\mathrm{CD} 8^{+} \mathrm{T}$ cells at the lymph nodes and suppressed the immune response [24]. It was speculated that exhausted $\mathrm{CD} 8^{+} \mathrm{T}$ cells gradually lost the ability to proliferate and expressed cytokines like interleukin-2 (IL-2), tumor necrosis factor-a (TNF-a), and interferon-gamma (IFN- $\gamma$ ) [25]. Moreover, an increased PD-1 expression was also observed on peripheral $\mathrm{CD} 4^{+} \mathrm{T}$ cells, while limited the extent of $\mathrm{CD}^{+} \mathrm{T}$ cell accumulation in response to an immunogenic stimulus [26]. And it strengthened the idea that PD-L1/PD-1 axis may play a role in osteosarcoma progression [20]. In our study, positive/higher PD-L1/PD-1 expression was associated with increased total mortality risk and decreased overall survival in osteosarcoma patients. The major mechanisms including immune tolerance and tumor progression may elucidate the poor prognosis of osteosarcoma with high PD-L1/PD-1 expression.

Due to its expression in both tumor cells and various immune cells, the PD-L1/PD-1 axis may be a promising target for immunotherapy of osteosarcoma [19]. According to the mechanisms mentioned above, reactivation of T cells by PD-L1/PD-1 blockade might increase the antitumor immune response [27]. Preclinical data demonstrated the benefit of combination therapy targeting both PD-L1 and CTLA-4 in a mouse model of metastatic osteosarcoma [28]. Furthermore, the efficacy of pembrolizumab (anti-PD-1 antibody) is being investigated for advanced sarcomas including osteosarcomas in the phase II SARC028 study (NCT02301039). In response to treatment with anti-PD-L1 antibody, tumor cells downregulated PD-L1 whereas upregulated CD80 and CD86. Meanwhile, improved CTLs function was also observed in vivo. Accordingly, PD-L1 monoclonal antibody was supposed to enhance T-cell-mediated rejection of metastatic osteosarcoma and potentially improve prognosis of patients [16]. However, due to the uncertain clinical efficacy and expensive cost of PD-L1 antibodies, additional therapeutic strategies targeting PD-L1 is warranted to treat osteosarcoma. Recent studies showed that PD-L1 sgRNA-guided CRISPR/Cas9 was able to specifically knockout PD-L1 expression [18]. The establishment of a constitutive PD-L1 knockout cell line provides unique insights into the application of CRISPR/Cas9 technology in osteosarcoma treatment.

Despite the promising data, some limitations still should be acknowledged. Firstly, the detecting and evaluating method of PD-L1/PD-1 expression in the eligible studies was not well defined. Differences in primary antibodies with varying dilutions, staining protocols, evaluation standards, and cutoff values for high/positive expression may contribute to heterogeneity. Secondly, the number of subjects in the included studies is relatively small, which might result in a lack of statistical power and prevent a meaningful analysis of the results. With the updating of gene chip and microarray platform technology and an explosion of PD-1/PD-L1 axis research in osteosarcoma, a significant extension of our finding and re-analysis, which will include more patients, could be accomplished in near future. Finally, when not reported in original articles, RRs were extrapolated from the survival curves or calculated from the provided data according to the method of Parmar et al. [29], which could introduce potential source of bias.

The present meta-analysis suggests that PD-L1/PD-1 overexpression in osteosarcoma was significantly associated with increased metastasis, with a tendency for higher total mortality risk and poorer overall survival. Accordingly, positive/higher PD-L1/PD-1 expression may be a significant biomarker for poor prognosis and the adverse clinicopathologic features of osteosarcoma. Additionally, the PD-L1/PD-1 axis may be a promising target for immunotherapy of osteosarcoma. Nevertheless, large-scale, multicenter and better-designed trials are warranted to further identify the clinicopathological and prognostic significance of PD-L1/PD-1 overexpression in osteosarcoma.

\section{Supplementary Material}

Supplementary figures.

http://www.jcancer.org/v09p2525s1.pdf

\section{Acknowledgements}

This work is supported by the National Key Research and Development Program of China (2016YFC1100100) and the Major Research Plan of National Natural Science Foundation of China (No. 91649204).

The views expressed in the submitted article are our own and not an official position of the institution or funder.

\section{Competing Interests}

The authors have declared that no competing interest exists.

\section{References}

1. Luetke A, Meyers PA, Lewis I, Juergens H. Osteosarcoma treatment - where do we stand? A state of the art review. Cancer Treat Rev. 2014; 40: 523-32.

2. Moore DD, Luu HH. Osteosarcoma. Cancer Treat Res. 2014: 65-92. 
3. Cui S, Su X, Dong L, Qian J, Ye L, Zhang T, et al. Programmed cell death ligand 1 protein levels predicted survival of non-small cell lung cancer. Journal of Cancer. 2017; 8: 4075-82.

4. Que Y, Xiao W, Guan YX, Liang Y, Yan SM, Chen HY, et al. PD-L1 Expression Is Associated with FOXP3+Regulatory T-Cell Infiltration of Soft Tissue Sarcoma and Poor Patient Prognosis. Journal of Cancer. 2017; 8: 2018-25.

5. Botti G, Scognamiglio G, Marra L, Pizzolorusso A, Di Bonito M, De Cecio R, et al. Programmed Death Ligand 1 (PD-L1) Expression in Primary Angiosarcoma. Journal of Cancer. 2017; 8: 3166-72.

6. Keir ME, Francisco LM, Sharpe AH. PD-1 and its ligands in T-cell immunity. Curr Opin Immunol. 2007; 19: 309-14.

7. Chen LP, Han X. Anti-PD-1/PD-L1 therapy of human cancer: past, present, and future. J Clin Invest. 2015; 125: 3384-91.

8. Meng XJ, Huang ZQ, Teng FF, Xing LG, Yu JM. Predictive biomarkers in PD-1/PD-L1 checkpoint blockade immunotherapy. Cancer Treat Rev. 2015; 41: 868-76.

9. Nomi T, Sho M, Akahori T, Hamada K, Kubo A, Kanehiro H, et al. Clinical significance and therapeutic potential of the programmed death-1 ligand/programmed death-1 pathway in human pancreatic cancer. Clin Cancer Res. 2007; 13: 2151-7.

10. Cao LJ, Wang XY, Li SY, Zhi OJ, Wang YQ, Wang LC, et al. PD-L1 is a Prognostic Biomarker in Resected NSCLC Patients with Moderate/high Smoking History and Elevated Serum SCCA Level. Journal of Cancer. 2017; 8: 3251-60

11. Topalian SL, Hodi FS, Brahmer JR, Gettinger SN, Smith DC, McDermott DF, et al. Safety, Activity, and Immune Correlates of Anti-PD-1 Antibody in Cancer. New Engl J Med. 2012; 366: 2443-54.

12. Romano E, Romero P. The therapeutic promise of disrupting the PD-1/PD-L1 immune checkpoint in cancer: unleashing the CD8 T cell mediated anti-tumor activity results in significant, unprecedented clinical efficacy in various solid tumors. J Immunother Cancer. 2015; 3.

13. Egger M, Smith GD, Schneider M, Minder C. Bias in meta-analysis detected by a simple, graphical test. Brit Med J. 1997; 315: 629-34.

14. Costa Arantes DA, Goncalves AS, Jham BC, Duarte ECB, de Paula EC, de Paula HM, et al. Evaluation of HLA-G, HLA-E, and PD-L1 proteins in oral osteosarcomas. Oral surgery, oral medicine, oral pathology and oral radiology. 2017; 123: e188-e96.

15. Sundara YT, Kostine $M$, Cleven AHG, Bovee JVMG, Schilham MW, Cleton-Jansen AM. Increased PD-L1 and T-cell infiltration in the presence of HLA class I expression in metastatic high-grade osteosarcoma: a rationale for T-cell-based immunotherapy. Cancer Immunol Immun. 2017; 66: 119-28.

16. Lussier DM, O'Neill L, Nieves LM, McAfee MS, Holechek SA, Collins AW, et al. Enhanced T-Cell Immunity to Osteosarcoma Through Antibody Blockade of PD-1/PD-L1 Interactions. J Immunother. 2015; 38: 96-106.

17. Shen JK, Cote GM, Choy E, Yang P, Harmon D, Schwab J, et al. Programmed Cell Death Ligand 1 Expression in Osteosarcoma. Cancer Immunol Res. 2014; 2: 690-8.

18. Liao YF, Chen LL, Feng $Y$, Shen J, Gao $Y$, Cote $G$, et al. Targeting programmed cell death ligand 1 by CRISPR/Cas9 in osteosarcoma cells. Oncotarget. 2017; 8: 30276-87.

19. Koirala P, Roth ME, Gill J, Piperdi S, Chinai JM, Geller DS, et al. Immune infiltration and PD-L1 expression in the tumor microenvironment are prognostic in osteosarcoma. Sci Rep-Uk. 2016; 6.

20. Zheng WJ, Xiao H, Liu H, Zhou Y. Expression of programmed death 1 is correlated with progression of osteosarcoma. Apmis. 2015; 123: 102-7.

21. Palmerini E, Agostinelli C, Picci P, Pileri S, Marafioti T, Lollini PL, et al. Tumoral immune-infiltrate (IF), PD-L1 expression and role of CD8/TIA-1 lymphocytes in localized osteosarcoma patients treated within protocol ISG-OS1. Oncotarget. 2017; 8: 111836-46.

22. Zou WP, Chen LP. Inhibitory B7-family molecules in the tumour microenvironment. Nat Rev Immunol. 2008; 8: 467-77.

23. Ramsay AG. Immune checkpoint blockade immunotherapy to activate anti-tumour T-cell immunity. Brit J Haematol. 2013; 162: 313-25.

24. Teng MWL, Ngiow SF, Ribas A, Smyth MJ. Classifying Cancers Based on T-cell Infiltration and PD-L1. Cancer Res. 2015; 75: 2139-45.

25. Ott PA, Hodi FS, Robert C. CTLA-4 and PD-1/PD-L1 Blockade: New Immunotherapeutic Modalities with Durable Clinical Benefit in Melanoma Patients. Clin Cancer Res. 2013; 19: 5300-9.

26. Okazaki T, Chikuma S, Iwai Y, Fagarasan S, Honjo T. A rheostat for immune responses: the unique properties of PD- 1 and their advantages for clinical application. Nat Immunol. 2013; 14: 1212-8.

27. Zhu ZH, Jin Z, Zhang M, Tang YJ, Yang G, Yuan XW, et al. Prognostic value of programmed death-ligand 1 in sarcoma: a meta-analysis. Oncotarget. 2017; 8: 59570-80.

28. Lussier DM, Johnson JL, Hingorani P, Blattman JN. Combination immunotherapy with alpha-CTLA-4 and alpha-PD-L1 antibody blockade prevents immune escape and leads to complete control of metastatic osteosarcoma. J Immunother Cancer. 2015; 3.

29. Parmar $\mathrm{MKB}$, Torri V, Stewart L. Extracting summary statistics to perform meta-analyses of the published literature for survival endpoints. Stat Med. 1998; 17: 2815-34. 